\title{
Effect of Nitrogen Management through Decision Support Tools on Nutrient Uptake, Efficiency and Economics in Direct Seeded Rice (Oryza sativa L.) under Tungabhadra Project (TBP) Command Area of Karnataka, India
}

\author{
Gurupadappa, S. R. Anand", M.R. Umesh, Y.M. Ramesha and J. Vishwanath \\ Department of Agronomy, University of Agricultural Sciences, Raichur (UASR), \\ Karnataka, India \\ *Corresponding author
}

\begin{tabular}{|l|}
\hline K e y w o r d s \\
$\begin{array}{l}\text { DSR, Decision support } \\
\text { tools, Economics, } \\
\text { Nitrogen management }\end{array}$ \\
\hline Article Info \\
\hline $\begin{array}{l}\text { Accepted: } \\
\text { 24 October } 2018 \\
\text { Available Online: } \\
\text { 10 December } 2018\end{array}$ \\
\hline
\end{tabular}

\section{Introduction}

Rice (Oryza sativa L.) is the staple food for more than half of the world's population. Worldwide, rice is grown in $161 \mathrm{~m}$ ha, with an annual production of about $678.7 \mathrm{mt}$ of paddy. About 90 per cent of the world's rice is grown and produced $(143 \mathrm{~m}$ ha of area with a
A B S T R A C T

A field experiment was conducted during kharif 2017 to study the "Nitrogen management through different decision support tools in Direct Seeded Rice (Oryza sativa L.) under Tungabhadra Project (TBP) command area of Karnataka" at Agricultural Research Station, Gangavathi. The soil type in the experimental site was medium black soil with soil $\mathrm{pH}$ and EC of 7.90 and 0.43 respectively. The initial soil available nitrogen, phosphorus and potassium was $213.2,31.05$ and $374.8 \mathrm{~kg} \mathrm{ha}^{-1}$, respectively which is in the range of low in nitrogen, medium in phosphorous and high in potassium content. The experiment consists of 12 treatments laid out in randomized block design with three replications. The results revealed that application of nitrogen through LCC $\leq 6.0$ threshold recorded significantly higher $\mathrm{N}, \mathrm{P}$ and $\mathrm{K}$ uptake at harvest $\left(184.2,35.2\right.$ and $137.8 \mathrm{~kg} \mathrm{ha}^{-1}$, respectively), which was on par with LCC $\leq 5.0$ threshold $\left(178.6,32.0\right.$ and $135.9 \mathrm{~kg} \mathrm{ha}^{-1}$, respectively), SPAD $\leq 40\left(164.0,30.6\right.$ and $134.6 \mathrm{~kg} \mathrm{ha}^{-1}$, respectively), SPAD $\leq 50(171.2,31.1$ and $135.1 \mathrm{~kg}$ $\mathrm{ha}^{-1}$, respectively), Green Seeker $\leq 0.8\left(156.7,30.1\right.$ and $133.8 \mathrm{~kg} \mathrm{ha}^{-1}$, respectively) as compared to $\operatorname{RDF}\left(93.2,24.1\right.$ and $125.4 \mathrm{~kg} \mathrm{ha}^{-1}$, respectively) and farmers practice (112.7, 25.8 and $129.6 \mathrm{~kg} \mathrm{ha}^{-1}$, respectively). Similar trend was also observed in economic. Whereas the application of nitrogen through LCC $\leq 4.0$ threshold recorded significantly higher Agronomic efficiency, Recovery efficiency and Partial factor productivity (38.57 $\mathrm{kg} \mathrm{kg}^{-1}, 98.33 \%$ and $76.73 \mathrm{~kg}$ grain $\mathrm{kg} \mathrm{N}^{-1}$, respectively), as compare to other treatments. Hence, precision nitrogen management in DSR can be done through LCC $\leq 5.0$ or SPAD $\leq$ 50 and green seeker $\leq 0.8$ threshold for obtaining nutrient uptake and economics. 
shortfall of rainfall every year resulting in declining resources. In TBP command area, cultivation of paddy is characterized by use of more $\mathrm{N}$ fertilizer without scientific base is the common practice besides unscientific water management followed by the formers of this command. In this context, management of $\mathrm{N}$ fertilizer to increase NUE and to avoid ground water contamination is need of the hour. IRRI Philippines introduced LCC is a hand held plastic strip, that can be used as a complementary decision making tool to determine the need for $\mathrm{N}$ application in field periodically. LCC has now been used successfully to guide fertilizer $\mathrm{N}$ application in rice, wheat and maize (Bijay Singh et al., 2002; Yadvinder Singh et al., 2007 and Varinderpal et al., 2010). The SPAD meter is a hand held, simple, quick and nondestructive in-situ tool for measuring relative content of chlorophyll in leaf that is directly proportional to leaf $\mathrm{N}$ content. Hence, the SPAD chlorophyll meter is used to diagnose the $\mathrm{N}$ status in crops and determine the right time of $\mathrm{N}$ application (Mohanty et al., 2016). The application of optical sensors in agriculture has advanced rapidly in the recent years. The Green seeker optical sensor works on reflection of light from the chlorophyll, similarly, these sensors use visible and nearinfrared (NIR) spectral radiation from plant canopies to detect $\mathrm{N}$ stress and crop vigour (NDVI) values are used as the basis for nitrogen application. NDVI measurements can range from -1 to 1 , with higher values indicating better plant health. It has the ability to predict yield potential of crops (Harrell et al., 2011). When we go for broad-based blanket recommendations of fertilizer $\mathrm{N}$ in field may end up with lower nutrient use efficiency because of large field-to field variability of soil $\mathrm{N}$, so Keeping in view the significance of $\mathrm{N}$ on productivity of rice, crop need based $\mathrm{N}$ fertilizer application through some of the decision support tools like LCC, SPAD and Green seeker will reduced the $\mathrm{N}$ losses, cost of fertilizer and application cost. Hence, there is urgent need to find out nitrogen threshold limit for direct seeded rice under TBP command area.

\section{Materials and Methods}

The field experiment was carried out at Agricultural Research Station, Gangavathi during kharif-2017 to study the "Nitrogen management through decision support tools in direct seeded rice (Oryza sativa L.) under Tungabhadra Project (TBP) command area." The experiment was replicated thrice in Randomized Complete Block Design. There were twelve treatment consisted of $\mathrm{T}_{1}-\mathrm{LCC} \leq$ 3.5 threshold, $\mathrm{T}_{2}-\mathrm{LCC} \leq 4.0$ threshold, $\mathrm{T}_{3}-$ LCC $\leq 4.5$ threshold, $\mathrm{T}_{4}-\mathrm{LCC} \leq 5.0$ threshold, $\mathrm{T}_{5}-\mathrm{LCC} \leq 6.0$ threshold, $\mathrm{T}_{6}-$ $\mathrm{SPAD} \leq 40, \mathrm{~T}_{7}-\mathrm{SPAD} \leq 50, \mathrm{~T}_{8}-$ Green Seeker $\leq 0.6, \mathrm{~T}_{9}$ - Green Seeker $\leq 0.8, \mathrm{~T}_{10}$ RDF (150:75:75 N: $\left.\mathrm{P}_{2} \mathrm{O}_{5}: \mathrm{K}_{2} \mathrm{O} \mathrm{kg} \mathrm{ha}^{-1}\right), \mathrm{T}_{11}-$ Farmers practice (200:100:66 N: $\mathrm{P}_{2} \mathrm{O}_{5}: \mathrm{K}_{2} \mathrm{O}$ $\left.\mathrm{kg} \mathrm{ha}^{-1}\right), \mathrm{T}_{12}$ - Absolute control. The soil was medium black with $\mathrm{pH}$ of 7.9 , EC (0.43 dS $\mathrm{m}^{-1}$ ), Organic carbon 0.46 and available Nitrogen, Phosphorous and Potassium (213.2. 31.05 and $374.8 \mathrm{~kg} \mathrm{ha}{ }^{-1}$, respectively). Nitrogen fertilizer apply based on LCC, SPAD and Green Seeker value recorded at 10 days interval (Table 1 and 2). The gross plot size was $6.0 \mathrm{~m} \times 6.0 \mathrm{~m}$ and net plot size was $5.2 \mathrm{~m}$ $\times 5.6 \mathrm{~m}$. Two seeds per hill were dibbled $5 \mathrm{~cm}$ deep in furrows at a spacing of $20 \mathrm{~cm} \mathrm{x} 10 \mathrm{~cm}$. Phosphorous and Potassium fertilizer was applied at 30 days while, nitrogen fertilizer was applied as per the treatments. The weed management was done through spraying of pre-emergent application pendimethaline 38.7 CS@ $2.5 \mathrm{ml} / \mathrm{l}$ of water followed by two hand weeding at 30 and 45 DAS. The crop was irrigated once in ten days before application of nitrogen fertilizer. Crop protection was done as per package of practice. Growth observations were recorded 30, 60, 90 DAS and at harvest. The yield observations were 
recorded at time of harvest. The crop was harvested at its physiological maturity. The data was statistically analyzed as per the procedure given by Gomez (1972).

\section{Results and Discussion}

Nitrogen uptake by plant at 30, 60, 90 DAS and harvest

The data on nitrogen uptake by plant at 30, 60, 90 DAS and at harvest as influenced by nitrogen management is presented in Table 3 . The results revealed that application of nitrogen through LCC $\leq 6.0$ threshold recorded significantly higher nitrogen uptake at 30, 60, 90 DAS and harvest (19.7, 80.0, 153.2 and $184.2 \mathrm{~kg} \mathrm{ha}^{-1}$, respectively), which was on par with LCC $\leq 5.0$ threshold (16.5, $75.1,148.3$ and $178.6 \mathrm{~kg} \mathrm{ha}^{-1}$, respectively), SPAD $\leq 40(15.4,65.3,136.6$ and $164.0 \mathrm{~kg}$ $\mathrm{ha}^{-1}$, respectively), SPAD $\leq 50$ (16.4, 70.7, 141.6 and $171.2 \mathrm{~kg} \mathrm{ha}^{-1}$, respectively), Green Seeker $\leq 0.8(13.6,60.5,127.6$ and $156.7 \mathrm{~kg}$ $\mathrm{ha}^{-1}$, respectively) as compared to RDF (8.9, $29.9,73.6$ and $93.2 \mathrm{~kg} \mathrm{ha}^{-1}$, respectively) and farmers practice $(10.2,39.6,89.2$ and $112.7 \mathrm{~kg}$ $\mathrm{ha}^{-1}$, respectively). Whereas, absolute control recorded significantly smaller plants compare to other treatments (Table 3).

\section{Major nutrient uptake by plant after harvest}

The data on major nutrient uptake by plant after harvest as influenced by nitrogen management is presented in Table 4. The results revealed that application of nitrogen through $\mathrm{LCC} \leq 6.0$ threshold recorded significantly higher $\mathrm{N}, \mathrm{P}$ and $\mathrm{K}$ uptake at harvest (184.2, 35.2 and $137.8 \mathrm{~kg} \mathrm{ha}^{-1}$, respectively), which was on par with $\mathrm{LCC} \leq$ 5.0 threshold (178.6, 32.0 and $135.9 \mathrm{~kg} \mathrm{ha}^{-1}$, respectively), SPAD $\leq 40$ (164.0, 30.6 and $134.6 \mathrm{~kg} \mathrm{ha}{ }^{-1}$, respectively), SPAD $\leq 50$ (171.2, 31.1 and $135.1 \mathrm{~kg} \mathrm{ha}^{-1}$, respectively),
Green Seeker $\leq 0.8(156.7,30.1$ and $133.8 \mathrm{~kg}$ $\mathrm{ha}^{-1}$, respectively) as compared to RDF (93.2, 24.1 and $125.4 \mathrm{~kg} \mathrm{ha}^{-1}$, respectively) and farmers practice $(112.7,25.8$ and $129.6 \mathrm{~kg}$ $\mathrm{ha}^{-1}$, respectively). Whereas, absolute control recorded significantly lower nutrient uptake compare to other treatments (Table 4). The growth and yield of rice is determined by the presence of sufficient quantity of nutrient in soil in the available form for plant uptake (Babannavar, 1990). The quantity of $\mathrm{N}$ uptake was directly related with the quantity of $\mathrm{N}$ applied to the soil. In LCC $\leq 6.0, \mathrm{LCC} \leq 5.0$, $\mathrm{SPAD} \leq 50, \mathrm{SPAD} \leq 40$ and Green Seeker $\leq$ 0.8 showed higher $\mathrm{N}$ uptake $(97.6,91.6,83.6$, 75.9 and $68.1 \%$, respectively) over RDF and farmers practice. This might be due to the uptake of $\mathrm{P}$ and $\mathrm{K}$ was directly proportional with the $\mathrm{N}$ uptake which proved the synchrony between $\mathrm{N}$ demand and supply from soil and fertilizer was probably the cause of increased uptake of $\mathrm{N}, \mathrm{P}$ and $\mathrm{K}$ (Dobermann et al., 2002). Increase in uptake of nutrient in LCC $\leq$ 6.0 and $\mathrm{SPAD} \leq 50$ treatments might be due to synergistic interaction between major nutrients and to the root as reported by Saikia and Pathak (1997).

\section{Efficiency indices}

The data on efficiency indices as influenced by nitrogen management is presented in Table 5. The results revealed that application of nitrogen through LCC $\leq 4.0$ threshold recorded significantly higher Agronomic efficiency, Recovery efficiency and Partial factor productivity $\left(38.57 \mathrm{~kg} \mathrm{~kg}^{-1}, 98.33 \%\right.$ and $76.73 \mathrm{~kg}$ grain $\mathrm{kg} \mathrm{N}^{-1}$, respectively), as compare to other treatments. Physiological efficiency is significantly higher with LCC $\leq$ 3.5 (90.16). Whereas, recovery efficiency of $\mathrm{LCC} \leq 4.0$ was on par with $\mathrm{LCC} \leq 6.0, \mathrm{LCC} \leq$ 5.0 and $\mathrm{SPAD} \leq 40$ (77.22, 88.93 and $83.64 \%$, respectively, table 5). The practice of balanced fertilization, one nutrient increases the efficiency of other through synergistic effect. 
Table.1 LCC, SPAD and green seeker values before N fertilizer application

\begin{tabular}{|c|c|c|c|c|c|c|c|c|c|c|}
\hline \multirow[t]{3}{*}{ Tr. No. } & \multirow[t]{3}{*}{ Treatments } & \multirow{3}{*}{$\begin{array}{l}\text { Basal dose } \\
\left(\mathrm{kg} \mathrm{N} \mathrm{ha}^{-1}\right)\end{array}$} & \multicolumn{8}{|c|}{ Decision support tools values } \\
\hline & & & \multicolumn{8}{|c|}{10 days interval (30 DAS) } \\
\hline & & & $10 / 9$ & $20 / 9$ & $30 / 9$ & $10 / 10$ & 20/10 & $30 / 10$ & 10/11 & Total \\
\hline $\mathbf{T}_{1}$ & LCC $\leq 3.5$ threshold & - & 2.00 & 2.90 & 3.75 & 4.00 & 4.20 & 4.30 & 4.20 & 60 \\
\hline $\mathbf{T}_{2}$ & LCC $\leq 4.0$ threshold & - & 2.40 & 3.10 & 4.15 & 4.20 & 4.25 & 4.30 & 4.20 & 60 \\
\hline $\mathbf{T}_{3}$ & LCC $\leq 4.5$ threshold & - & 2.20 & 3.20 & 4.00 & 4.40 & 4.59 & 4.65 & 4.55 & 120 \\
\hline $\mathbf{T}_{4}$ & LCC $\leq 5.0$ threshold & - & 2.50 & 3.00 & 4.20 & 4.50 & 4.85 & 5.20 & 5.00 & 150 \\
\hline $\mathbf{T}_{5}$ & LCC $\leq 6.0$ threshold & - & 2.60 & 3.50 & 4.45 & 5.25 & 5.75 & 6.05 & 5.80 & 180 \\
\hline $\mathbf{T}_{6}$ & $\mathrm{SPAD} \leq 40$ & - & 26.8 & 32.1 & 35.5 & 38.7 & 42.5 & 43.5 & 38.9 & 150 \\
\hline $\mathbf{T}_{7}$ & $\mathrm{SPAD} \leq 50$ & - & 27.2 & 33.2 & 37.6 & 42.9 & 46.8 & 48.4 & 51.1 & 180 \\
\hline $\mathbf{T}_{8}$ & Green Seeker $\leq 0.6$ & - & 0.31 & 0.41 & 0.50 & 0.58 & 0.65 & 0.64 & 0.59 & 150 \\
\hline$T_{9}$ & Green Seeker $\leq 0.8$ & - & 0.32 & 0.46 & 0.57 & 0.68 & 0.76 & 0.83 & 0.78 & 180 \\
\hline $\mathbf{T}_{10}$ & RDF (150:75:75) & 75 & - & 20 & - & - & 30 & - & 30 & 150 \\
\hline $\mathbf{T}_{11}$ & $\begin{array}{l}\text { Farmers } \\
\text { practice(200:100:66) }\end{array}$ & 100 & - & - & - & - & 55 & - & - & 200 \\
\hline $\mathbf{T}_{12}$ & Absolute control & - & - & - & - & - & - & - & - & - \\
\hline
\end{tabular}

Note - RDF: (150:75:75 N: $\left.\mathrm{P}_{2} \mathrm{O}_{5}: \mathrm{K}_{2} \mathrm{O} \mathrm{kg} \mathrm{ha}{ }^{-1}\right)$ and Farmers practice: $\left(200: 100: 66 \mathrm{~N}: \mathrm{P}_{2} \mathrm{O}_{5}: \mathrm{K}_{2} \mathrm{O} \mathrm{kg} \mathrm{ha}{ }^{-1}\right)$. 
Table.2 Quantity of $\mathrm{N}$ fertilizer applied in different treatments

\begin{tabular}{|c|c|c|c|c|c|c|c|c|c|c|}
\hline \multirow[t]{3}{*}{ Tr. No. } & \multirow[t]{3}{*}{ Treatments } & \multirow{3}{*}{$\begin{array}{l}\text { Basal dose } \\
\left(\mathrm{kg} \mathrm{N} \mathrm{ha}^{-1}\right)\end{array}$} & \multicolumn{8}{|c|}{ Top dress } \\
\hline & & & \multicolumn{8}{|c|}{10 days interval $\left(\mathrm{kg} \mathrm{N} \mathrm{ha}^{-1}\right)$} \\
\hline & & & $10 / 9$ & $20 / 9$ & $30 / 9$ & $10 / 10$ & 20/10 & 30/10 & 10/11 & Total \\
\hline $\mathbf{T}_{1}$ & LCC $\leq 3.5$ threshold & - & 30 & 30 & - & - & - & - & - & 60 \\
\hline $\mathbf{T}_{2}$ & LCC $\leq 4.0$ threshold & - & 30 & 30 & - & - & - & - & - & 60 \\
\hline $\mathbf{T}_{3}$ & LCC $\leq 4.5$ threshold & - & 30 & 30 & 30 & 30 & - & - & - & 120 \\
\hline $\mathbf{T}_{4}$ & LCC $\leq 5.0$ threshold & - & 30 & 30 & 30 & 30 & 30 & - & - & 150 \\
\hline $\mathbf{T}_{5}$ & LCC $\leq 6.0$ threshold & - & 30 & 30 & 30 & 30 & 30 & - & 30 & 180 \\
\hline $\mathrm{T}_{6}$ & $\mathrm{SPAD} \leq 40$ & - & 30 & 30 & 30 & 30 & - & - & 30 & 150 \\
\hline $\mathbf{T}_{7}$ & $\mathrm{SPAD} \leq 50$ & - & 30 & 30 & 30 & 30 & 30 & 30 & - & 180 \\
\hline $\mathbf{T}_{8}$ & Green Seeker $\leq 0.6$ & - & 30 & 30 & 30 & 30 & - & - & 30 & 150 \\
\hline $\mathbf{T}_{9}$ & Green Seeker $\leq 0.8$ & - & 30 & 30 & 30 & 30 & 30 & - & 30 & 180 \\
\hline $\mathbf{T}_{10}$ & RDF (150:75:75) & 75 & - & 20 & - & - & 30 & - & 30 & 150 \\
\hline $\mathbf{T}_{11}$ & Farmers practice(200:100:66) & 100 & - & - & - & - & 55 & - & - & 200 \\
\hline $\mathbf{T}_{12}$ & Absolute control & - & - & - & - & - & - & - & - & - \\
\hline
\end{tabular}

Note - RDF: (150:75:75 N: $\left.\mathrm{P}_{2} \mathrm{O}_{5}: \mathrm{K}_{2} \mathrm{O} \mathrm{kg} \mathrm{ha}{ }^{-1}\right)$ - Basal $\left(75 \mathrm{Kg} \mathrm{N}^{-1}{ }^{-1}\right)$ and 2 Split $\left(37.5 \mathrm{Kg} \mathrm{N}^{-1}\right)$ at 60, 90 DAS each.

Farmers practice: (200:100:66 N: $\left.\mathrm{P}_{2} \mathrm{O}_{5}: \mathrm{K}_{2} \mathrm{O} \mathrm{kg} \mathrm{ha}{ }^{-1}\right)$ - Basal $\left(100 \mathrm{Kg} \mathrm{N}^{-1}\right)$ and 2 Split $\left(50 \mathrm{Kg} \mathrm{N}^{-1}\right)$ at 60, $90 \mathrm{DAS}$ each. 
Table.3 Nitrogen uptake $\left(\mathrm{kg} \mathrm{ha}^{-1}\right)$ by plant at 30, 60, 90 DAS and harvest of direct seeded rice as influenced by $\mathrm{N}$ management through different decision support tools

\begin{tabular}{|c|c|c|c|c|c|}
\hline & & $\begin{array}{c}\text { Nitrogen uptake } \\
\text { at } 30 \text { DAS } \\
\left(\mathrm{kg} \mathrm{ha}^{-1}\right)\end{array}$ & $\begin{array}{c}\text { Nitrogen uptake } \\
\text { at } 60 \text { DAS } \\
\left(\mathrm{kg} \mathrm{ha}^{-1}\right)\end{array}$ & $\begin{array}{c}\text { Nitrogen uptake } \\
\text { at } 90 \text { DAS } \\
\left(\mathrm{kg} \mathrm{ha}^{-1}\right)\end{array}$ & $\begin{array}{c}\text { Nitrogen uptake } \\
\text { at harvest } \\
\left(\mathrm{kg} \mathrm{ha}^{-1}\right)\end{array}$ \\
\hline $\mathbf{T}_{1}$ & LCC $\leq 3.5$ threshold & 7.7 & 24.2 & 56.5 & 66.4 \\
\hline $\mathbf{T}_{2}$ & LCC $\leq 4.0$ threshold & 9.6 & 34.1 & 80.6 & 104.2 \\
\hline $\mathbf{T}_{3}$ & LCC $\leq 4.5$ threshold & 12.8 & 45.4 & 101.1 & 125.3 \\
\hline $\mathbf{T}_{4}$ & LCC $\leq 5.0$ threshold & 16.5 & 75.1 & 148.3 & 178.6 \\
\hline $\mathbf{T}_{5}$ & LCC $\leq 6.0$ threshold & 19.7 & 80.0 & 153.2 & 184.2 \\
\hline $\mathrm{T}_{6}$ & $\mathrm{SPAD} \leq 40$ & 15.4 & 65.3 & 136.6 & 164.0 \\
\hline $\mathbf{T}_{7}$ & $\mathrm{SPAD} \leq 50$ & 16.4 & 70.7 & 141.6 & 171.2 \\
\hline $\mathbf{T}_{8}$ & Green Seeker $\leq 0.6$ & 11.3 & 43.2 & 95.1 & 119.2 \\
\hline $\mathbf{T}_{9}$ & Green Seeker $\leq 0.8$ & 13.6 & 60.5 & 127.6 & 156.7 \\
\hline $\mathbf{T}_{10}$ & $\operatorname{RDF}\left(150: 75: 75 \mathrm{~N}: \mathrm{P}_{2} \mathrm{O}_{5}: \mathrm{K}_{2} \mathrm{O} \mathrm{kg} \mathrm{ha}{ }^{-1}\right)$ & 8.9 & 29.9 & 73.6 & 93.2 \\
\hline $\mathbf{T}_{11}$ & Farmers practice(200:100:66 N: $\left.\mathrm{P}_{2} \mathrm{O}_{5}: \mathrm{K}_{2} \mathrm{O} \mathrm{kg} \mathrm{ha}^{-1}\right)$ & 10.2 & 39.6 & 89.2 & 112.7 \\
\hline $\mathrm{T}_{12}$ & Absolute control & 5.5 & 16.2 & 39.4 & 45.2 \\
\hline & S. Em. \pm & 2.2 & 7.3 & 8.6 & 9.4 \\
\hline & C.D. at $5 \%$ & 6.4 & 21.6 & 25.9 & 28.1 \\
\hline
\end{tabular}


Table.4 Quantity of $\mathrm{N}\left(\mathrm{kg} \mathrm{ha}^{-1}\right)$ applied and major nutrient uptake $\left(\mathrm{kg} \mathrm{ha}^{-1}\right)$ by plant after harvest in direct seeded rice as influenced by $\mathrm{N}$ management through different decision support tools

\begin{tabular}{|c|c|c|c|c|c|}
\hline \multicolumn{2}{|r|}{ Treatments } & $\begin{array}{c}\text { N applied (kg } \\
\left.\text { ha }^{-1}\right)\end{array}$ & $\begin{array}{l}\text { Nitrogen } \\
\left(\mathrm{kg} \mathrm{ha}^{-1}\right)\end{array}$ & $\begin{array}{l}\text { Phosphorus } \\
\qquad\left(\mathrm{kg} \mathrm{ha}^{-1}\right)\end{array}$ & $\begin{array}{l}\text { Potassium } \\
\left(\mathrm{kg} \mathrm{ha}^{-1}\right)\end{array}$ \\
\hline $\mathbf{T}_{1}$ & LCC $\leq 3.5$ threshold & 60.00 & 66.4 & 23.2 & 126.8 \\
\hline $\mathbf{T}_{2}$ & LCC $\leq 4.0$ threshold & 60.00 & 104.2 & 24.7 & 127.5 \\
\hline $\mathbf{T}_{3}$ & $\mathrm{LCC} \leq 4.5$ threshold & 120.00 & 125.3 & 28.9 & 132.2 \\
\hline $\mathbf{T}_{4}$ & LCC $\leq 5.0$ threshold & 150.00 & 178.6 & 32.0 & 135.9 \\
\hline $\mathbf{T}_{5}$ & LCC $\leq 6.0$ threshold & 180.00 & 184.2 & 32.5 & 137.8 \\
\hline$T_{6}$ & $\mathrm{SPAD} \leq 40$ & 150.00 & 164.0 & 30.6 & 134.6 \\
\hline $\mathbf{T}_{7}$ & $\mathrm{SPAD} \leq 50$ & 180.00 & 171.2 & 31.1 & 135.1 \\
\hline $\mathrm{T}_{8}$ & Green Seeker $\leq 0.6$ & 150.00 & 119.2 & 27.5 & 130.4 \\
\hline $\mathbf{T}_{9}$ & Green Seeker $\leq 0.8$ & 180.00 & 156.7 & 30.1 & 133.8 \\
\hline $\mathrm{T}_{10}$ & $\operatorname{RDF}\left(150: 75: 75 \mathrm{~N}: \mathrm{P}_{2} \mathrm{O}_{5}: \mathrm{K}_{2} \mathrm{O} \mathrm{kg} \mathrm{ha}{ }^{-1}\right)$ & 150.00 & 93.2 & 24.1 & 125.4 \\
\hline $\mathbf{T}_{11}$ & Farmers practice (200:100:66 N: $\left.\mathrm{P}_{2} \mathrm{O}_{5}: \mathrm{K}_{2} \mathrm{O} \mathrm{kg} \mathrm{ha}^{-1}\right)$ & 200.00 & 112.7 & 25.8 & 129.6 \\
\hline $\mathbf{T}_{12}$ & Absolute control & 00.00 & 45.2 & 21.0 & 118.7 \\
\hline & S. Em. \pm & & 9.4 & 0.89 & 1.35 \\
\hline & C.D. at $\mathbf{5 \%}$ & & 28.1 & 2.61 & 4.00 \\
\hline
\end{tabular}




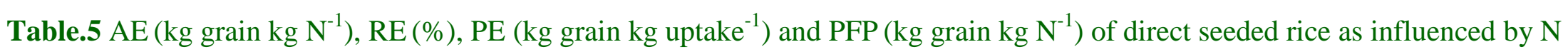
management through different decision support tools

\begin{tabular}{|c|c|c|c|c|c|}
\hline \multicolumn{2}{|r|}{ Treatments } & \multirow{2}{*}{$\begin{array}{c}\text { AE } \\
\left(\mathbf{k g}_{\text {grain }} \mathbf{k g ~ N}^{-\mathbf{1}}\right) \\
26.04\end{array}$} & \multirow{2}{*}{$\begin{array}{l}\text { RE } \\
(\%) \\
35.33\end{array}$} & \multirow{2}{*}{$\begin{array}{c}\text { PE } \\
\left(\mathrm{kg} \mathrm{kg}^{-\mathbf{1}}\right) \\
90.16\end{array}$} & \multirow{2}{*}{ 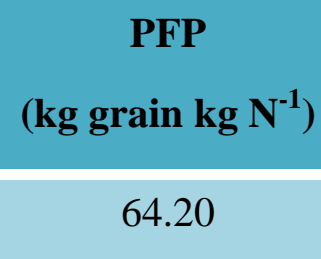 } \\
\hline $\mathbf{T}_{1}$ & LCC $\leq 3.5$ threshold & & & & \\
\hline $\mathbf{T}_{2}$ & LCC $\leq 4.0$ threshold & 38.57 & 98.33 & 38.96 & 76.73 \\
\hline $\mathbf{T}_{3}$ & LCC $\leq 4.5$ threshold & 25.23 & 66.75 & 38.85 & 44.31 \\
\hline $\mathbf{T}_{4}$ & LCC $\leq 5.0$ threshold & 25.78 & 88.93 & 30.41 & 41.04 \\
\hline $\mathbf{T}_{5}$ & LCC $\leq 6.0$ threshold & 21.89 & 77.22 & 30.15 & 34.61 \\
\hline $\mathbf{T}_{6}$ & $\mathrm{SPAD} \leq 40$ & 22.89 & 83.64 & 28.16 & 38.15 \\
\hline $\mathbf{T}_{7}$ & $\mathrm{SPAD} \leq 50$ & 19.83 & 70.00 & 31.15 & 32.55 \\
\hline $\mathbf{T}_{8}$ & Green Seeker $\leq 0.6$ & 19.87 & 49.33 & 40.02 & 35.13 \\
\hline $\mathbf{T}_{9}$ & Green Seeker $\leq 0.8$ & 17.84 & 61.94 & 29.49 & 30.56 \\
\hline $\mathbf{T}_{10}$ & RDF (150:75:75 N: $\left.\mathrm{P}_{2} \mathrm{O}_{5}: \mathrm{K}_{2} \mathrm{O} \mathrm{kg} \mathrm{ha}{ }^{-1}\right)$ & 14.14 & 32.00 & 44.55 & 29.40 \\
\hline $\mathbf{T}_{11}$ & Farmers practice(200:100:66 N: $\mathrm{P}_{2} \mathrm{O}_{5}: \mathrm{K}_{2} \mathrm{O} \mathrm{kg} \mathrm{ha}^{-1}$ ) & 13.98 & 33.75 & 41.36 & 25.43 \\
\hline & S. Em. \pm & 2.27 & 7.20 & 11.59 & 2.64 \\
\hline & C.D. at $5 \%$ & 6.66 & 21.12 & 33.99 & 7.75 \\
\hline
\end{tabular}

Note: AE-Agronomic efficiency, RE- Recovery efficiency, PE- Physiological efficiency and PFP- partial factor productivity. 
Table.6 Cost of cultivation, Gross returns, Net returns and Benefit cost ratio of direct seeded rice as influenced by $\mathrm{N}$ management through different decision support tools

\begin{tabular}{|c|c|c|c|c|c|}
\hline \multicolumn{2}{|r|}{ Treatments } & \multirow{2}{*}{$\begin{array}{c}\text { Cost of cultivation } \\
\qquad \begin{array}{c}\left.\text { (Rs. ha }^{-\mathbf{1}}\right) \\
41950\end{array} \\
\end{array}$} & \multirow{2}{*}{$\begin{array}{c}\text { Gross returns } \\
\begin{array}{c}(\text { Rs. ha } \\
\text {-1) }\end{array} \\
63685\end{array}$} & \multirow{2}{*}{$\begin{array}{c}\text { Net returns } \\
\text { (Rs. ha }^{-1} \text { ) } \\
21735\end{array}$} & \multirow{2}{*}{$\begin{array}{c}\text { Benefit cost } \\
\text { ratio } \\
1.52\end{array}$} \\
\hline $\mathbf{T}_{1}$ & LCC $\leq 3.5$ threshold & & & & \\
\hline $\mathbf{T}_{2}$ & LCC $\leq 4.0$ threshold & 41950 & 76099 & 34149 & 1.81 \\
\hline $\mathbf{T}_{\mathbf{3}}$ & LCC $\leq 4.5$ threshold & 42733 & 89533 & 46800 & 2.10 \\
\hline $\mathbf{T}_{4}$ & LCC $\leq 5.0$ threshold & 43125 & 101618 & 58233 & 2.34 \\
\hline $\mathbf{T}_{5}$ & LCC $\leq 6.0$ threshold & 43515 & 102847 & 59332 & 2.36 \\
\hline $\mathbf{T}_{6}$ & $\mathrm{SPAD} \leq 40$ & 43124 & 94515 & 51391 & 2.19 \\
\hline $\mathbf{T}_{7}$ & $\mathrm{SPAD} \leq 50$ & 43516 & 96801 & 53285 & 2.22 \\
\hline $\mathbf{T}_{8}$ & Green Seeker $\leq 0.6$ & 43125 & 87148 & 44023 & 2.02 \\
\hline $\mathbf{T}_{9}$ & Green Seeker $\leq 0.8$ & 43516 & 90900 & 47384 & 2.09 \\
\hline $\mathbf{T}_{10}$ & $\mathrm{RDF}\left(150: 75: 75 \mathrm{~N}: \mathrm{P}_{2} \mathrm{O}_{5}: \mathrm{K}_{2} \mathrm{O} \mathrm{kg} \mathrm{ha}^{-1}\right)$ & 42740 & 82422 & 39682 & 1.92 \\
\hline $\mathbf{T}_{11}$ & Farmers practice(200:100:66 N: $\left.\mathrm{P}_{2} \mathrm{O}_{5}: \mathrm{K}_{2} \mathrm{O} \mathrm{kg} \mathrm{ha}^{-1}\right)$ & 44321 & 87341 & 43020 & 1.97 \\
\hline $\mathbf{T}_{12}$ & Absolute control & 36000 & 37965 & 1965 & 1.05 \\
\hline \multicolumn{3}{|c|}{ S. Em. \pm} & 4300 & 4300 & 0.10 \\
\hline \multicolumn{3}{|c|}{ C.D. at $5 \%$} & 12614 & 12614 & 0.29 \\
\hline
\end{tabular}


In the present study, application of $\mathrm{N}$ through LCC, SPAD and Green Seeker recorded higher AE, RE and PFP over the RDF and farmers' practice. The $\mathrm{LCC} \leq 4.0, \mathrm{LCC} \leq 3.5$, $\mathrm{LCC} \leq 5.0, \mathrm{LCC} \leq 6.0$ and $\mathrm{SPAD} \leq 40$ have recorded a higher $\mathrm{AE}(175.0,86.2,84.4,56.5$ and $63.7 \%$, respectively), RE (191.3, 4.6, $163.4,128.8$ and $147.8 \%$, respectively) and PFP (201, 154.7, 61.3, 36.0 and 50.0\%, respectively) over farmer's method. Whereas, $\mathrm{PE}$ was higher in RDF compare to LCC, SPAD and Green Seeker. The split application of N using LCC, SPAD and Green Seeker resulted in increased leaf $N$ concentration which might have promoted photosynthetic efficiency, better assimilation of photosynthates toward grain and adequate quantity of $\mathrm{N}$ during critical stages of plant growth resulted in better growth characters and yield components at various phenological stages. These results are confined with the findings of Dobermann et al., (2000), Stalin et $a l$. , (2008) and Shukla (2004).

\section{Economics}

Gross returns, net returns and $\mathrm{B}: \mathrm{C}$ ratio was significantly higher with application of nitrogen through LCC $\leq 6.0$ threshold (102847, 59332 Rs. $\mathrm{ha}^{-1}$ and 2.36, respectively), which was on par with $\mathrm{LCC} \leq$ 5.0 threshold (101618, 58233 and 2.34, respectively), SPAD $\leq 40(94515,51391$ and 2.19, respectively), SPAD $\leq 50$ (96801, 53285 and 2.22, respectively), Green Seeker $\leq$ 0.8 (90900, 47384 and 2.09, respectively) as compared to RDF (82422, 39682 and 1.92, respectively) and farmers practice (87341, 43020 and 1.97, respectively). Whereas, absolute control recorded significantly lower net returns compare to other treatments (Table 6). Increase in $\mathrm{LCC} \leq 3.5$ to $\mathrm{LCC} \leq 6.0$ increased the net returns to a tune of 172.9 per cent. Similarly, the per cent increase in net returns in $\mathrm{LCC} \leq 5.0, \mathrm{SPAD} \leq 40$, and Green Seeker $\leq 0.8(167.9 \%, 136.4 \%$ and $118.0 \%$, respectively). The benefit cost ratio was higher at $\mathrm{LCC} \leq 6.0$ (2.36), $\mathrm{LCC} \leq 5.0$ (2.34), $\mathrm{SPAD} \leq 40$ (2.19), $\mathrm{SPAD} \leq 50$ (2.22) and Green Seeker $\leq 0.8$ (2.09) over RDF and farmers practice, indicating LCC $\leq 5.0$ and $\mathrm{SPAD} \leq 40$ could be the optimum level. The above results are in line with the findings of Balasubramanian et al., (2000) and Mallikarjuna et al., (2016) who opined that need-based application of $\mathrm{N}$ fertilizer following LCC, SPAD and Green Seeker revealed substantial gains to farmers through reduction of $\mathrm{N}$ and insecticides use, and a small increase in grain yields and income over farmers' practice.

Among the different decision support tools, threshold value of $\mathrm{LCC} \leq 6.0$, recorded higher nutrient uptake and economics as compare to other treatments. However, there was much difference in the uptake of nutrient and economics between LCC $\leq 6.0$ and 5.0, SPAD $\leq 40$ and 50. Hence, LCC $\leq 5.0$ and $\mathrm{SPAD} \leq 40$ was found optimum for DSR in TBP command area as evidenced in higher net returns and $\mathrm{B}$ : $\mathrm{C}$ ratio.

\section{References}

Babannavar, P. B., 1990, Response of sunflower (Halianthus annus L.) to the varying levels of nitrogen and phosphorus under saline water irrigation. M.Sc. (Agri) Thesis, Univ. Agric. Sci., Dharwad, Karnataka, India.

Balasubramanian, V., Morales, A. C., Cruz, R. T., Thiyagarajan, T. M., Nagarajan, R., Babu, M., Abdulrachman, S. and Hai, L. H., 2000, Application of the chlorophyll meter (SPAD) technology for real-time $\mathrm{N}$ management in rice. A review, Int. Rice Res., Insti. NEWS Notes, 251:4-8.

Bijay Singh, Yadvinder Singh, Jagadish, K. L., Kevin, F., Bronson, Vethaiya Ala Subramanian, Jagdeep Singh, Charan, 
S. and Khind, 2002, Chlorophyll meter and LCC based nitrogen management for rice and wheat in Northern India. Agron. J., 94: 821-829.

Dobermann, A. and Fairhurst, T., 2000, Rice nutrient disorders and nutrient management. Potash and phosphate institute of Canada and Int. Rice Res. notes., Singapore and Los Banos, pp.191.

Dobermann, A., Witt, C., Dawe, D., Gines, H. C., Nagarajan, R., Satawathananont, S., Son, T. T., Tan, P. S., Wang, G. H., Chien, N. V., Thoa, V. T. K., Phung, C. V., Stalin, P., Muthukrishnan, P., Ravi, V., Babu, M., Chatuporn, S., Kongchum, M., Sun, Q., Fu, R., Simbahan, G. C. and Adviento, M. A. A., 2002. Site-specific nutrient management for intensive rice cropping systems in Asia, Field Crops Res., 74: $37-66$.

Gomez, K. A., 1972, Layout, sampling, sources of error technologies for field experiment with rice, Int. Rice Res. Insti. Notes., Los Bonos, Philippines, pp. 37-39.

Harrell, D. L., Tubana, B. S., Walker, T. S. and Phillips, S. B., 2011, Estimating rice grain yield potential using normalized difference vegetation index. Agron. J., 103: 1717-1723.

Mallikarjuna Swamy, Umesh, M. R., Ananda, N., Shanwad, U. K., Amaregouda, A. and Manjunath, N., 2016, Precision nitrogen management for rabi sweet corn (Zea mays saccharata L.) through decision support tools. J. Farm Sci., 29(1):14-18.

Mohanty, S. K., Jat, S. L., Parihar, C. M., Singh, A. K., Sharma, S., Saveipune, D., Sandhya and Kuri, B. R., 2016, Precision nitrogen management practices in wheat influences nutrient uptake and their use efficiencies and fertility status of soil under conservation agriculture. Ann. Agric. Res. 37(3): 282289.

Saikia, J. K. and Pathak, A. K., 1997, Effect of levels of NPK with or without soil test on grain yield of low land kharif rice and its residual effect on preceding summer rice. Annals Agric. Res., 18(4): $530-532$.

Shukla, A. K., Ladha, J. K., Singh, V. K., Dwivedi, B. S., Balasubramanian, V., Gupta, R. K., Sharma, S. K., Singh, Y., Pathay, H., Pandey, P. S. and Yadav, R. L., 2004, Calibrating leaf colour chart for nitrogen management in different genotypes of rice and wheat in a system perspective. Agron. J., 96: 1606-1621.

Varinderpal, S. and Yadvinder, S., 2010, Calibrating the leaf colour chart and chlorophyll meter for need based fertilizer nitrogen management in rice and wheat. Field Crops Res., 117: 154161

Yadvinder Singh, Bijay Singh, Ladha, J. K., Bains, J. S., Gupta, R. K., Jagmohan Singh, R. K. and Balasubramanian. V., 2007, Nutrient Cycling in Agroecosystem, 78: 167-176.

\section{How to cite this article:}

Gurupadappa, S.R. Anand, M.R. Umesh, Y.M. Ramesha and Vishwanath, J. 2018. Effect of Nitrogen Management through Decision Support Tools on Nutrient Uptake, Efficiency and Economics in Direct Seeded Rice (Oryza sativa L.) under Tungabhadra Project (TBP) Command Area of Karnataka, India. Int.J.Curr.Microbiol.App.Sci. 7(12): 3400-3410. doi: https://doi.org/10.20546/ijcmas.2018.712.389 